\title{
Inserzione del catetere peritoneale: esperienza personale
}

\author{
A. Favazza, G. Boscutti, G. Mioni \\ Divisione di Nefrologia e Dialisi - Ospedale Civile di Udine
}

ei pazienti in dialisi peritoneale le complicanze infettive e meccaniche legate al catetere peritoneale (CP) sono la principale causa di morbilità, perdita del CP e passaggio all'emodialisi (1). Per tale motivo sono state proposte varie modificazioni dei cateteri e delle tecniche di impianto. Attualmente i $\mathrm{CP}$ più diffusi sono il tradizionale catetere di Tenckhoff ed il catetere "a collo di cigno" ("Swan neck"), ideato da Twardowski (2) (Fig.1). Quest'ultimo si caratterizza per la presenza di una curva tra le due cuffie, per cui i 2 segmenti terminali, a CP inserito, sono rivolti in basso. Tale configurazione riduce il rischio di dislocazione dell'estremità intraperitoneale e dei conseguenti problemi di drenaggio (3). Secondo alcuni, il CP di "Swan neck", grazie all'exit site (foro cutaneo di uscita del $\mathrm{CP}$ ) rivolto caudalmente, ha anche il vantaggio di impedire il ristagno delle secrezioni cutanee circostanti. Nei casi di infezione, inoltre, un foro cutaneo rivolto caudalmente consentirebbe il deflusso delle secrezioni purulente, favorendo quindi la guarigione e riducendo il rischio delle infezioni secondarie del tunnel (che corrisponde al percorso del CP nello spessore della parete addominale, tra l'ingresso nella cavità peritoneale e l'exit site) e del peritoneo $(2,4)$. Per tali motivi lo stesso Tenckhoff nel 1968 proponeva di inseri- re il $\mathrm{CP}$ in un tunnel sottocutaneo ricurvo, concavo caudalmente (5). Talvolta sono stati utilizzati anche dei tunnellizzatori ricurvi, simili a quelli utilizzati dai chirughi per inserire i drenaggi sottocutanei. Ma l'esperienza ha dimostrato che se si posiziona il tradizionale CP di Tenckhoff in un tunnel ricurvo, esso tende progressivamente a raddrizzarsi, per cui l'exit site diventa indirizzato lateralmente (con eventuale fuoriuscita della cuffia esterna) e/o l'estremità interna tende a dislocarsi, con conseguenti problemi di drenaggio (2). Alla luce di tali considerazioni, coloro che ritengono vantaggioso un $\mathrm{CP}$ che abbia le 2 estremità rivolte caudalmente, abitualmente utilizzano il CP di "Swan neck". Ma tale $\mathrm{CP}$ ha un costo superiore rispetto al $\mathrm{CP}$ di Tenckhoff, ed il suo inserimento richiede la preparazione di un tunnel sottocutaneo che riproduca esattamente la forma e le dimensioni del CP.

Nel nostro reparto dal 1988 utilizziamo, per il posizionamento del tradizionale $\mathrm{CP}$ di Tenckhoff, con 1 o 2 cuffie, un tunnellizzatore semicircolare da noi progettato. Esso ha lo scopo di inserire il segmento extraperitoneale del $\mathrm{CP}$ in un tunnel che, oltre ad essere ricurvo e concavo caudalmente, sia al contempo in grado di impedire il raddrizzamento del CP.

Il tunnellizzatore (TUN) è di acciaio inox ed ha una forma semicircolare, con un raggio di curvatura di 4,5 $\mathrm{cm}$ (Fig. 2). L'estremità anteriore è appuntita ed il diametro aumenta quindi gradualmente fino ad eguagliare il diametro esterno del CP. L'estremità posteriore ha diametro

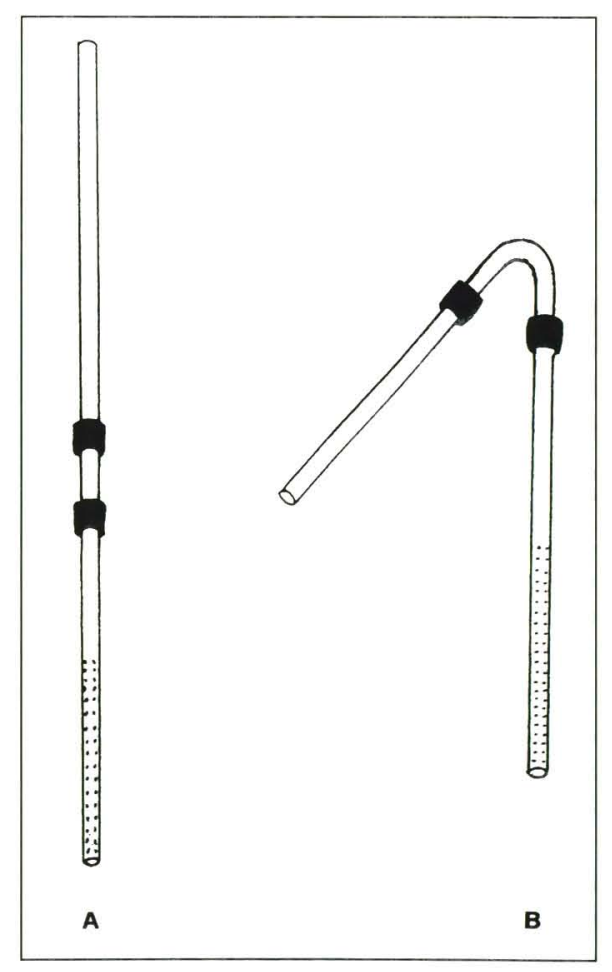

Fig. 1

$a$ - Catetere di Tenckhoff

b-Catetere "Swan neck" 


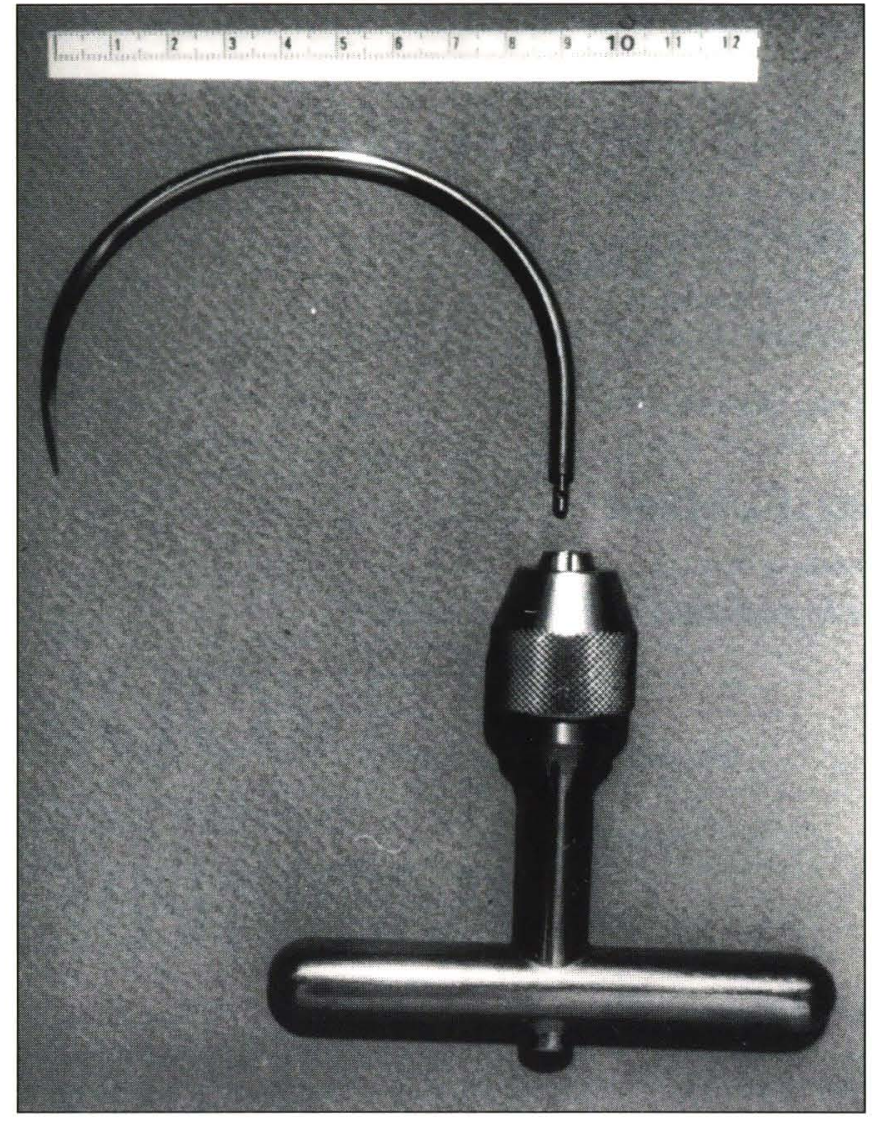

Fig. 2

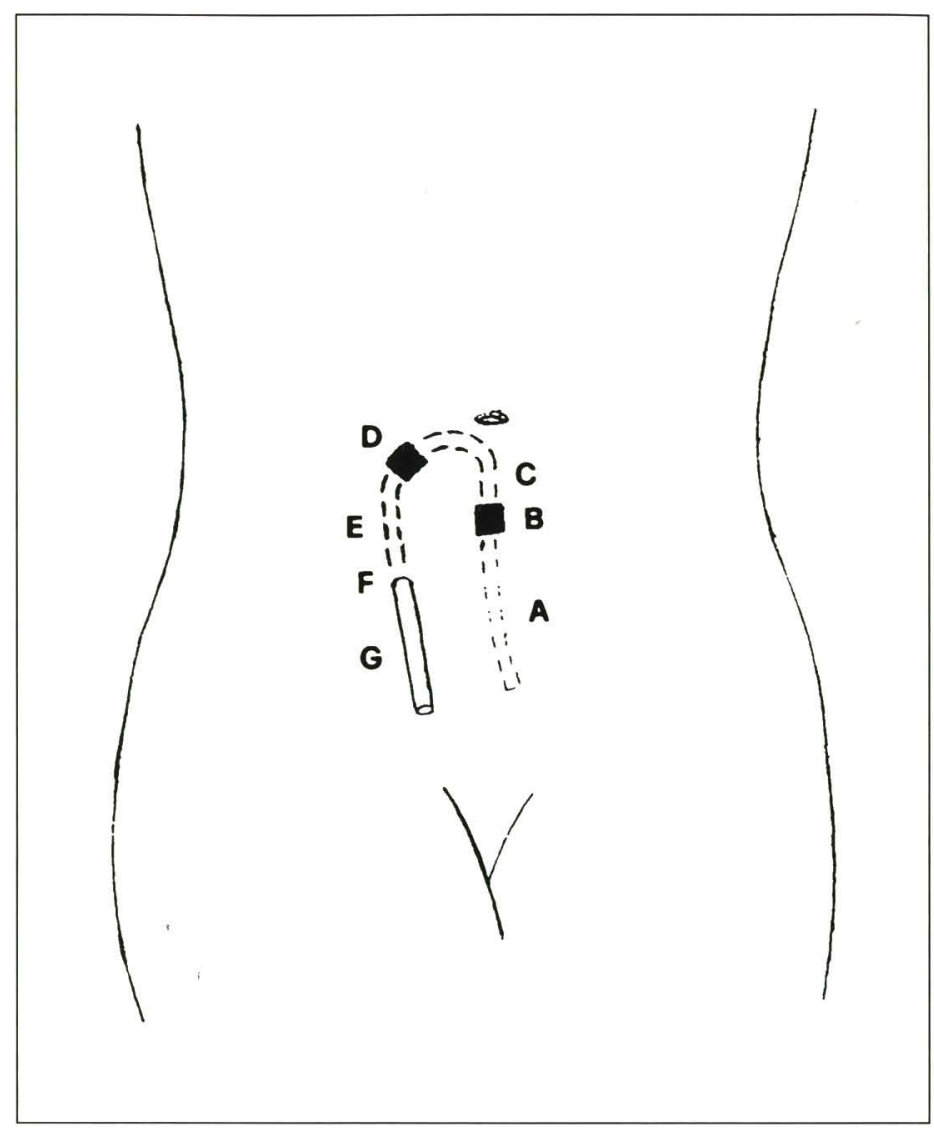

Fig. 3 ridotto per la lunghezza di $1 \mathrm{~cm}$, con un piccolo foro trasversale.

Per introdurre il CP nella cavità peritoneale abbiamo preferito adottare la tecnica chirurgica, cioè l'inserimento del CP previo isolamento ed apertura del peritoneo parietale. Grazie alla disponibilità "didattica" di un chirurgo, abbiamo acquisito nell' arco di qualche mese la capacità di effettuare in modo autonomo tale intervento, limitando il supporto del chirurgo solo ai reinterventi o ai pazienti con complicanze addominali.

Il posizionamento del $\mathrm{CP}$ venne sempre effettuato in anestesia locale, dopo la somministrazione di una singola dose di antibiotico (di solito ceftriaxone). L'incisione cutanea e la successiva dissezione degli strati sottostanti furono effettuati sulla linea mediana, e non attraverso il ventre del muscolo retto, come si fa abitualmente. Dopo che il CP fu introdotto nella cavità peritoneale, il peritoneo fu chiuso con sutura a borsa di tabacco attorno alla cuffia profonda, laddove usualmente la cuffia profonda rimane ex- traperitoneale e quindi il peritoneo viene suturato direttamente sul CP. Subito all'esterno della borsa di tabacco, il CP fu indirizzato cranialmente per una lunghezza di circa $2-3 \mathrm{~cm}$ e quindi fatto fuoriuscire dalla fascia del muscolo retto attraverso un ristretto spazio tra i 2 punti di sutura più craniali applicati ad essa. Tramite una impugnatura (Fig. 2) il TUN fu quindi introdotto perpendicolarmente nel margine dx della ferita chirurgica, nello strato profondo del tessuto sottocutaneo, e dopo una rotazione di circa 90110 gradi fu diretto caudalmente ed un po' medialmente, fino a perforare la cute (previamente anestetizzata) all'incirca 5$6 \mathrm{~cm}$ dalla linea mediana e $2-3 \mathrm{~cm}$ sotto il livello della cuffia profonda. L'estremità appuntita del TUN ha consentito di non utilizzare mai il bisturi per incidere la cute, per cui il foro cutaneo fu sempre ben aderente al CP fin dal momento del suo inserimento. L'impugnatura fu quindi rimossa e l'estremità posteriore del TUN, avente un diametro ridotto, fu inserita dentro l'estremità esterna del CP e fissata temporaneamente ad essa tramite una legatura passante attraverso il foro esistente nell'estremità posteriore del TUN. Il TUN fu quindi interamente tirato fuori dalla cute, trascinando in tal modo lungo il tunnel da esso formato il CP. Con tale metodica, il catetere assume all'incirca la forma di una U capovolta, schematizzata nella Figura 3, in cui:

$\mathrm{A}=$ segmento interno (intra-peritoneale) del catetere;

$\mathrm{B}=$ cuffia profonda;

$\mathrm{C}=$ segmento interno del tunnel (cioè sottofasciale, fissato in direzione craniocaudale tra i primi 2 dei punti di sutura applicati sulla fascia, cranialmente, e la sutura a borsa di tabacco del peritoneo attorno alla cuffia profonda, caudalmente); $\mathrm{D}=$ cuffia superficiale (presente solo nei cateteri con 2 cuffie);

$\mathrm{E}=$ segmento sottocutaneo del tunnel; $\mathrm{F}=$ foro di uscita del catetere (exit site); $\mathrm{G}=$ segmento esterno del catetere.

Va sottolineato che il tunnel ha una lunghezza di circa $10-13 \mathrm{~cm}$, mentre con le tecniche convenzionali esso è lungo 5-8 


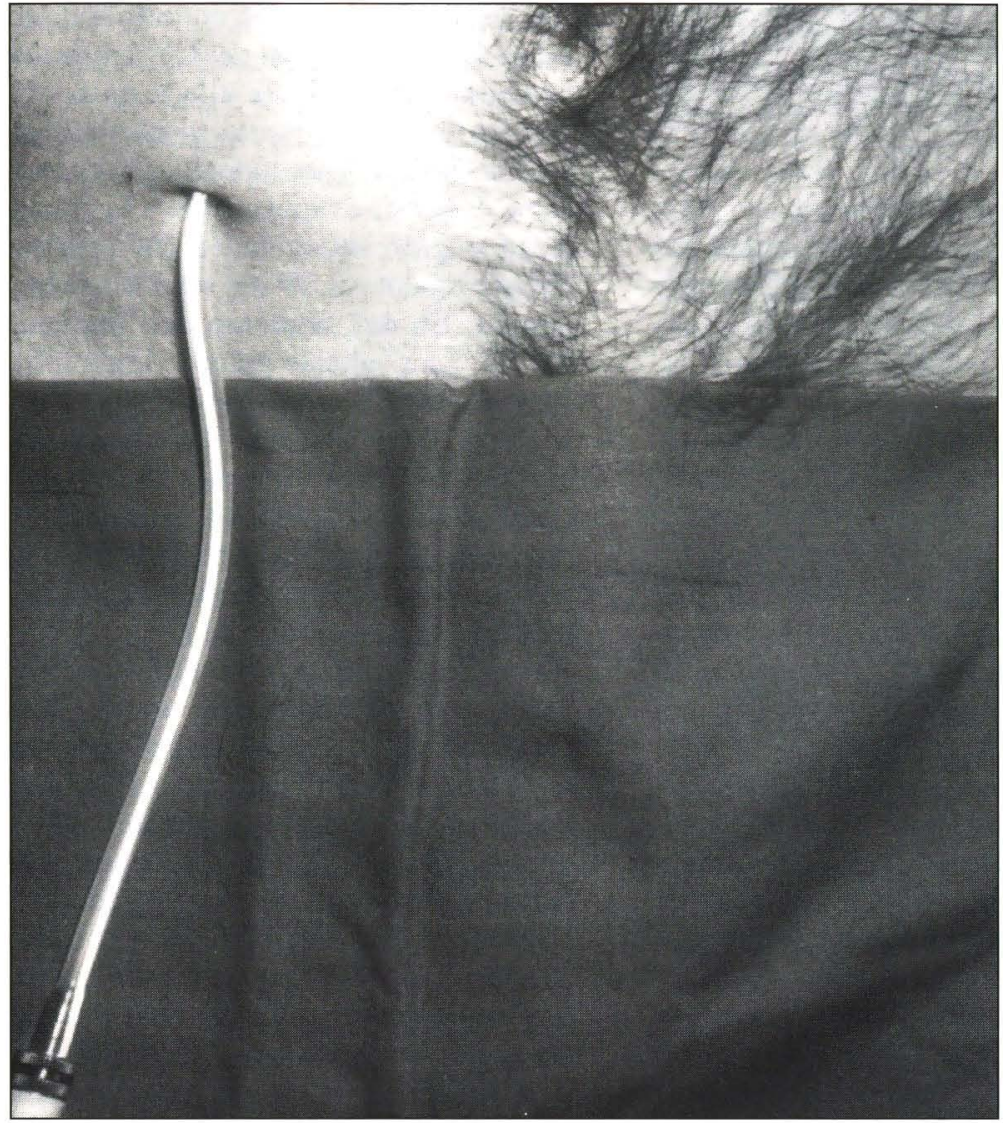

Fig. 4

TABELLA I - COMPLICANZE E CONSEGUENTI RIMOZIONI DEI CATETERI PERITONEALI $(\mathrm{CP})$

\begin{tabular}{|c|c|c|c|}
\hline & $\begin{array}{c}\text { Episodi } \\
\mathrm{N}^{\circ}\end{array}$ & Incidenza & $\begin{array}{c}\text { CP rimossi } \\
\mathrm{N}^{\circ}(\%)\end{array}$ \\
\hline Infezioni dell'emergenza cutanea (ESI) & 119 & 1 epis. ogni 33 mesi & $3(2)$ \\
\hline Infezioni del tunnel (IT) & 21 & 1 epis. ogni 185 mesi & $5(3.4)$ \\
\hline Peritoniti & 120 & 1 epis. ogni 32 mesi & $19(12.8)$ \\
\hline Peritoniti associate ad ESI e/o IT & $13(11 \%)$ & - & $* *$ \\
\hline Difficoltà di drenaggio per dislocaz. & 15 & - & $4(2.7)$ \\
\hline Leakage & 4 & - & - \\
\hline Ernia incisionale & 4 & - & $1(0.7)$ \\
\hline
\end{tabular}

** I CP rimossi per peritonite secondaria ad ESI o IT furono considerati rimossi per ESI o IT.

$\mathrm{cm}$. Nei CP con 2 cuffie, la distanza della cuffia esterna dall'exit site è di circa $3-5 \mathrm{~cm}$, laddove abitualmente è di $2 \mathrm{~cm}$. La Figura 4 evidenzia l'exit site ed il segmento esterno del catetere, orientati caudalmente.

La medicazione dell'exit-site era effettuata con betadine, ogni 1-2 giorni.

Subito dopo l'inserimento del CP, e successivamente a giorni alterni, venivano introdotti nella cavità peritoneale, e drenati immediatamente dopo, 500-1000 cc di dialisato senza aggiunta di farmaci. Gli scambi peritoneali venivano iniziati abitualmente dopo 10 giorni dall'inserimento del CP.

\section{Risultati}

Da giugno 1988 ad agosto 1996, 149 CP (72 con 1 cuffia, 77 con 2 cuffie) furono posizionati in 149 pazienti (età $=65$ \pm 16 ) affetti da uremia terminale.
I CP inseriti dopo la rimozione di un precedente CP non sono stati considerati in questa casistica.

Il follow-up fu di mesi 3877, rispettivamente 1918 per i CP con 1 cuffia (media $=$ mesi $26.7 \pm 18), 1959$ per i $\mathrm{CP}$ con 2 cuffie (mesi $25.4 \pm 22$ ).

\section{Complicanze infettive}

Infezioni dell'exit site (ESI): 64 pazienti (43\%) hanno presentato 119 episodi (in media 1 episodio ogni 33 mesi). 39 (54\%) dei pazienti con 1 cuffia hanno manifestato 70 episodi (1 ogni 27 mesi); 25 (32\%) dei pazienti con 2 cuffie 49 episodi ( 1 ogni 40 mesi)(Chi-quadro: $p=0.012$ ). Gli episodi di ESI comparsi entro 40 giorni dall'intervento sono stati 7.

3 CP furono rimossi per ESI recidivante; 12 episodi di ESI si sono complicati con infezione del tunnel; 2 guarirono dopo la rimozione della cuffia esterna; gli altri episodi guarirono con terapia antibiotica locale e/o sistemica.

Infezioni del tunnel (IT): sono stati osservati 21 episodi ( 1 ogni 185 mesi), di cui 14 nei pazienti con 1 cuffia ( 1 ogni 137 mesi), e 7 nei pazienti con 2 cuffie ( 1 ogni 280 mesi) (Chi-quadro: $\mathrm{p}=0.16$ ). 5 episodi portarono alla rimozione del $\mathrm{CP}$; gli altri 16 guarirono con terapia antibiotica sistemica.

Peritoniti (Perit): gli episodi furono 120 (1 ogni 32 mesi), di cui 72 nei pazienti con 1 cuffia ( 1 ogni 27 mesi), e 48 nei pazienti con 2 cuffie (1 ogni 41 mesi) (Chi-quadro: $\mathrm{p}=0.47$ ). Le Perit secondarie ad ESI e/o IT (abbiamo definito tali le Perit comparse entro 4 settimane dalla sospensione del trattamento antibiotico) furono 13 (l'11\% del totale delle Perit). Gli episodi comparsi entro 40 giorni dall'inserimento del CP sono stati 4. I CP rimossi per Perit furono 19.

\section{Complicanze meccaniche}

15 pazienti manifestarono difficoltà di drenaggio secondarie a dislocazione dell'estremità intraperitoneale del $\mathrm{CP}$ al di fuori del piccolo bacino. In 5 di essi il controllo $\mathrm{Rx}$ ha evidenziato il raddrizzamento del CP e quindi l'assenza della conformazione arcuata del tunnel, mentre negli altri 10 tale conformazione per- 
sisteva. In 11 pazienti l'estremità interna del CP fu nuovamente indirizzata caudalmente con l'attivazione della peristalsi o tramite manipolazione endoluminale con una guida flessibile (6). I rimanenti $4 \mathrm{CP}$ furono rimossi.

In nessun CP ci fu l'esteriorizzazione della cuffia esterna.

In 4 pazienti $(2.7 \%)$ si manifestò leakage, cioè la fuoriuscita di dialisato dalla cavità peritoneale attorno al CP. Tale complicanza regredì dopo la sospensione degli scambi peritoneali per 8 giorni.

In 4 pazienti, tutte donne, si sviluppò una ernia incisionale, cioè nella zona di inserzione del CP. In 2 di essi fu necessario un intervento di plastica, che provocò la rimozione del CP in 1 paziente.

Le varie complicanze e le conseguenti rimozioni del CP sono elencate nella Tabella I.

La sopravvivenza cumulativa dei CP fu del $92 \%$ a 1 anno, $86 \%$ a $2,76 \%$ a 3 , $64 \%$ a $4,54 \%$ a 5 , considerando come evento finale dei CP la loro rimozione per tutte le complicanze (infettive e meccaniche) e per le peritoniti. La sopravvivenza cumulativa fu statisticamente simile per i CP con 1 cuffia e con 2 cuffie.

\section{Discussione}

Questa nostra esperienza è caratterizzata dall'utilizzo di un semplice TUN semicircolare allo scopo di inserire il CP di Tenckhoff, con 1 o 2 cuffie, in un tunnel avente una curvatura di circa $180^{\circ}$ concava caudalmente.

Per impedire il raddrizzamento del CP si è reso necessario qualche piccolo accorgimento nella tecnica chirurgica. Per imporre all'estremità interna, intraperitoneale, una stabile direzione caudale, un segmento del CP lungo circa $2-3 \mathrm{~cm}$, compreso tra la borsa di tabacco ed il passaggio attraverso la fascia del muscolo retto, è stato fissato in direzione cranio-caudale. Noi abbiamo osservato problemi di drenaggio secondari a dislocazione del CP al di fuori del piccolo bacino in 15 pazienti. Ma va sottolineato che solo in 5 di essi la dislocazione era secondaria al raddrizzamento del CP. La dislocazione degli altri $10 \mathrm{CP}$ era indipendente dalla nostra tecnica chirurgica, in quanto al controllo Rx il segmento interno del tunnel manteneva una direzione declive.

L'altra possibile conseguenza del rad- drizzamento del CP è la lateralizzazione dell'exit site, con l'eventuale esteriorizzazione della cuffia esterna. Per impedire tale complicanza, nella manovra di tunnellizzazione il TUN è stato indirizzato, oltre che caudalmente, un po' medialmente, e la cute è stata perforata circa $2 \mathrm{~cm}$ al di sotto del livello della cuffia profonda (Fig. 3). Adottando tale accorgimento, il foro cutaneo non si è mai lateralizzato.

Un'altra importante complicanza meccanica del catetere peritoneale è il leakage. Esso impone la sospensione degli scambi peritoneali e favorisce le complicanze infettive. Nei casi di inserimento del CP sulla linea mediana il leakage è stato riportato fino nel $20 \%$ dei casi, per cui di solito si attua l'impianto in sede paramediana, cioè a livello del muscolo retto (13). Pur con tale accorgimento, il leakage continua a rappresentare una complicanza piuttosto frequente. Noi abbiamo preferito l'inserimento del CP attraverso la linea mediana, certamente più semplice $\mathrm{e}$ con un minore rischio di sanguinamento. La molto ridotta incidenza di leakage nella nostra casistica $(2.7 \%)$ va attribuita probabilmente alla migliore tenuta della legatura del peritoneo attorno alla cuffia profonda, rispetto alla legatura sulla superficie liscia del CP, effettuata abitualmente. D'altronde non abbiamo mai osservato aderenze delle anse intestinali attorno alla cuffia né difficoltà chirurgiche nei casi di asportazione del CP.

Le infezioni del CP, cioè a carico dell'exit site e del tunnel, sono diventate la più importante causa di morbilità e di perdita del CP (7). In una grossa casistica, il $17 \%$ delle peritoniti era associato ad esse (8). Il $25 \%$ degli episodi di infezione del foro cutaneo, in uno studio (9), ed il $21 \%$ degli episodi di infezione del foro cutaneo e/o del tunnel, in un altro studio (8), hanno indotto la rimozione del CP. Dall'8 al 39\% dei CP sono stati rimossi a causa di infezione del foro $\mathrm{cu}$ taneo e/o del tunnel (10).

Certamente i confronti tra le varie casistiche si devono valutare con cautela, soprattutto perché sono numerosi i fattori che condizionano l'insorgenza e l'evoluzione delle complicanze infettive legate al CP. Comunque, la nostra esperienza si confronta favorevolmente con le casistiche citate prima. Va sottolineato soprattutto il ridotto numero dei CP rimossi per ESI ed IT, e delle peritoniti secondarie a tali infezioni. Riteniamo che questi posi- tivi risultati possano essere attribuiti sia all'emergenza cutanea del CP indirizzata caudalmente $(2,4)$, sia alla tecnica chirurgica da noi adottata. In particolare, l'utilizzazione di un tunnellizzatore consente di allestire rapidamente e facilmente un tragitto sottocutaneo che aderisce completamente al CP per tutta la sua lunghezza, fin dal momento dell'impianto del CP. La risultante precoce immobilizzazione del CP ed un foro cutaneo ben aderente al $\mathrm{CP}$ riducono il rischio di una colonizzazione batterica a livello dell'exit site nell'immediato periodo postoperatorio, colonizzazione batterica che ha una certa responsabilità nelle infezioni sia precoci sia tardive (11).

$\mathrm{Va}$ anche sottolineato che utilizzando un TUN semicircolare avente un raggio di curvatura simile a quello da noi progettato, il tunnel risulta lungo circa $10-13 \mathrm{~cm}$, laddove l'inserimento sia del CP di Tenckhoff sia del CP di "Swan neck" dà luogo abitualmente ad un tunnel di circa $5-8 \mathrm{~cm}$. Un tunnel lungo, e con il suo segmento distale rivolto caudalmente, molto probabilmente ostacola l'arrivo dei germi nel peritoneo, e giustifica il ridotto numero di peritoniti secondarie alle infezioni del CP osservato nella nostra casistica.

Infine, è interessante il riscontro di una incidenza statisticamente minore delle infezioni dell'exit site (e tendenzialmente delle infezioni del tunnel) nei $\mathrm{CP}$ con 2 cuffie rispetto ai $\mathrm{CP}$ con 1 cuffia. Come già segnalato, nei nostri pazienti la cuffia esterna, per quanto riguarda i CP con 2 cuffie, era situata in sede pittosto profonda, e cioè a circa $3-5 \mathrm{~cm}$ dal foro cutaneo, laddove usualmente essa viene posta a circa $2 \mathrm{~cm}$. La nostra esperienza suggerisce quindi che la cuffia sottocutanea, anche se situata profondamente, riduce il rischio delle infezioni dell'exit site, verosimilmente grazie ad una migliore immobilizzazione del CP. D'altronde una cuffia esterna più profonda presenta i vantaggi di un minor rischio sia di esteriorizzazione sia di coinvolgimento in caso di infezione del foro cutaneo. Quest'ultimo aspetto è clinicamente importante in quanto una cuffia infetta è spesso refrattaria al trattamento medico $(12,13)$.

In conclusione, la nostra esperienza suggerisce che con l'ausilio di un semplice e facilmente riproducibile tunnellizzatore semicircolare il CP standard di Tenckhoff può essere stabilmente posi- 
zionato in un tunnel ricurvo, con le 2 estremità rivolte caudalmente. La tecnica da noi adottata e tale conformazione del tunnel risultano vantaggiose ai fini di una buona sopravvivenza dei CP.

\section{BIBLIOGRAFIA}

1. Gokal R, Ash SR, Helfrich $\mathrm{GB}$, et al. Peritoneal catheters and exit-site practices: toward optimum peritoneal access. Perit Dial Bull 1993; 13: 29-39.

2. Twardowski ZJ, Nolph KD, Khanna $\mathrm{R}$, et al. The need for a "swan neck" permanently bent, arcuate peritoneal dialysis catheter. Perit Dial Bull 1985; 5: 219-23.

3. Di Paolo N, Capotondo L, Garosi G, Guarnieri A. Cateteri peritoneali e tecniche di inserzione. In: Di Paolo N, ed. CAPD. Editoriale Bios; 1991: 155-86.

4. Copley JB. Prevention of peritoneal dialysis catheter-related infections. Am J Kidney Dis 1987; 10: 401-7.

5. Tenckhoff H, Schechter H. A bacteriologically safe peritoneal access device. ASAIO Trans 1968; 14: 181-6.

6.

Acciarri P, Cicoli L, Micucci G, Coppola E, Riggio S, Lombardo V. Dislocazione del catetere peritoneale. Nuova tecnica di riposizionamento (Rapporto tecnico). Giornale Italiano di Nefrologia 1992; 9: 357 63.

7. Burkart JM. Significance, epidemiology, and prevention of peritoneal catheter infections. Perit Dial Int 1996; (suppl)1: S340-6.

8. Piraino B, Bernardini J, Sorkin M. A five-year study of the microbiologic results of exit site infections and peritonitis in Continuous Ambulatory Peritoneal Dialysis. Am J Kidney Dis 1987; 10: 281-6.

9.

Scalamogna A, Castelnovo C, De Vecchi A, Ponticelli C. Exit-site and tunnel infections in Continuous Ambulatory Peritoneal Dialysis patients. Am J Kidney Dis 1991; 18: 674-7.

10. Lindblad AS, Hamilton RW, Nolph KD, Novak JW. A retrospec- tive analysis of catheter configuration and cuff type: A National CAPD Registry Report. Perit Dial Int 1988; 8: 129-33.

11. Twardowski ZJ, Prowant BF. Exit-site healing post catheter implantation. Perit Dial Int 1996; (suppl)3: S51-68.

12. Khanna R, Oreopoulos DG. Peritoneal catheters. In:Bengmark $\mathrm{S}$, ed. The peritoneum and peritoneal access. London: Wright; 1989: 220-9.

13. Nebel M, Marczewski K, Finke K. Three years of experience with the Swan-Neck Tenckhoff Catheter. In: Khanna R, Nolph KD, et al, eds. Advances in Peritoneal Dialysis. Nashville; 1991: 208-13. 